Luís Machado - University of Coimbra, Portugal

Graça Simões - University of Coimbra, Portugal

Claudio Gnoli - University of Pavia, Italy

Renato Souza - Getulio Vargas Foundation, Brazil

\title{
Can an Ontologically-Oriented KO Do Without Concepts?
}

\begin{abstract}
:
The ontological approach in the development of KOS is an attempt to overcome the limitations of the traditional epistemological approach. Questions raise about the representation and organization of ontologically-oriented $\mathrm{KO}$ units, such as BFO universals or ILC phenomena. The study aims to compare the ontological approaches of BFO and ILC using a hermeneutic approach. We found that the differences between the units of the two systems are primarily due to the formal level of abstraction of BFO and the different organizations, namely the grouping of phenomena into ILC classes that represent complex compounds of entities in the BFO approach. In both systems the use of concepts is considered instrumental, although in the ILC they constitute the intersubjective component of the phenomena whereas in BFO they serve to access the entities of reality but are not part of them.
\end{abstract}

\subsection{The aim and scope of the study}

In the literature associated with the Knowledge Organization area two approaches are found, the ontological one and the epistemological one, that are often associated with antagonistic positions, sometimes extreme (objectivism and subjectivism). Several authors, e.g.: Ridi (2016); Kleineberg (2017); Dahlberg (2017); Hjørland (2010), claim the lack of grounds for such positions, emphasizing that, in procedural terms, knowledge is both ontological and epistemological. As an area of study, ontology focuses on how reality is constituted and structured using concepts. However, even ontology uses concepts, the formation of which falls within the scope of epistemology, which studies how knowledge is acquired and grounded. Therefore, as Poli and Obrst (2010) state, the procedures of these areas complement and condition each other.

Seeking to overcome the inadequacy of the traditional epistemological approach in the development of knowledge organization systems (KOS) to interdisciplinary research, several authors argue ${ }^{1}$ that: "the basic units of the new KOS should be phenomena of the real world." This turn towards an ontological approach raises questions about the representation and organization of these new units.

Based on an ontological realism, Arp et al. (2015) consider that ontologies shouldn't focus on concepts but on universals or types (bringing together common characteristics of instantiated particulars). Within this theoretical position Klein and Smith (2010) distinguish concept systems - as systems of meanings; from representation systems which relate to real world entities. This is the approach of Basic Formal Ontology (BFO), an upper-ontology that bridges ontologies from different domains, recently adopted as an information exchange standard (ISO/IEC CD 21838-2).

${ }^{1}$ During the 8th conference of the ISKO Spanish chapter (2017), as part of the proposals labeled as "the León manifesto." http://www.iskoi.org/ilc/leon.php (access on 8.1.2020). 
Apparently in a seemingly position of compromise, Gnoli (2018) includes concepts, as produced through the evolving interaction of percepts (individual experience of reality) and previously accumulated knowledge, in the notion of phenomena, that are taken as the basic units of the Integrative Levels Classification (ILC). The combinable facets of ILC include several "elements of perspective" seeking to integrate epistemological aspects into an ontological KOS as noted by Kleineberg (2020). This feature aims to overcome the limits of the disciplinary compartmentalization of KOSs such as the Dewey Decimal Classification or the Universal Decimal Classification which, in addition, present some misfit to the digital context as stated by Szostak et al. (2016); Dahlberg (2017); Simões et al. (2016); Gnoli (2008).

The aim of this paper is to compare the ontological approaches of the BFO and the ILC, by identifying the elementary units that constitute them and the guiding principles that underlie their organization. We discussed the preliminary results of an ongoing $\mathrm{PhD}$ research on this topic, hoping that our study will bring useful contributions, such as the systematization of a set of guidelines for ontological KOS modeling. We consider these contributions as being useful both in the scope of the bibliographic classification systems, where the ontological approach has less tradition, and in the scope of the computational ontologies where, as Machado et al. (2020) state, "a careful intervention can be the distinguishing mark of the KO and LIS areas," regarding a greater intervention of researchers from these areas in the development of this type of KOS.

\subsection{The method}

Methodologically, a comparison method will be employed using the hermeneutic approach complemented with content analysis methods following Bardin (2011) and Kuckartz (2014). The methodological complementary allows a more in-depth analysis of the literature that applies extended segments, the units of context, as a way to facilitate the understanding of the core units of analysis, the units of meaning.

\subsection{ILC and BFO units}

ILC (2nd edition) units - the phenomena apprehended from reality, are related in six levels (strata) of formal dependence (information; matter; life; mind; societies; and works). These are divided into layers that are materially dependent on each other (e.g., the life stratum has, in order of dependence, four layers: genes; bacteria; eukaryote organisms; populations). The layers (25 in total) form the main classes of the ILC which, in turn, contain subclasses in variable numbers (e.g., fungi, plants and animals are some subclasses of organisms). Subclasses are ordered (whenever possible) by the principle of evolutionary appearance (emergence), each forming its hierarchical chain of types and subtypes (e.g., animals $>$ chordates $>$ mammals $>$ whales).

It is in this latter form of organization, in a taxonomy of types and subtypes that the $\mathrm{BFO}$ units - the entities of the real world, are structured. Being a formal ontology (in the Husserlian sense of being neutral to any domain) its units are very general types of entities. The first division is between entities that continue or persist through time (continuant) and entities that unfolds themselves in time (occurrent). In BFO 2.0 continuant has three subtypes: independent continuant (with two subtypes of entity: material and immaterial); specifically dependent continuant (also with two subtypes: quality and realizable entity) and generically dependent continuant. As subtypes of 
occurrent BFO presents four: process; process boundary; temporal region and spatiotemporal region.

Given the not entirely taxonomic structure of ILC, mapping between ILC layers of phenomena and BFO entity types should be understood as a mere academic exercise for the comparative study of its ontological structures. An attempt to distribute the ILC layers across the three main types of BFO entity (independent continuant, dependent continuant and occurrent) is shown in Table 1. In this comparison we do not include the ILC layer forms, belonging to the information strata, since this class deals with mathematical entities and, according to Smith et al. (2015), BFO 2.0 does not deal with those entities, although they are partially addressed in lower level ontologies built on BFO. $^{2}$

Table 1: Exploratory distribution of ILC classes by types of entities at the top-level of the BFO

\begin{tabular}{|c|c|c|c|}
\hline ILC strata & \multicolumn{3}{|c|}{ ILC layers } \\
\hline information & & & spacetime; branes \\
\hline matter & $\begin{array}{l}\text { energy; atoms; molecules; } \\
\text { celestial bodies; rocks; land }\end{array}$ & continuum bodies & \\
\hline life & $\begin{array}{l}\text { genes; bacteria; organisms; } \\
\text { populations }\end{array}$ & & \\
\hline $\operatorname{mind}$ & & instincts; consciousness & \\
\hline societies & & $\begin{array}{l}\text { language; polities; } \\
\text { technologies; rituals; } \\
\text { communities; enterprises }\end{array}$ & \\
\hline works & artifacts & knowledge; artworks & \\
\hline & BFO independent continuant & BFO dependent continuant & BFO occurrent \\
\hline
\end{tabular}

The option for the highest level's types of BFO units is due, on the one hand, to the inclusion, in some ILC classes, of phenomena that may be considered entities of different types at more BFO specific levels and, on the other hand, due to the ontological complexity of some phenomena involved. Still, for some classes the relationship of the respective phenomena to more specific types of BFO entities appears to be less intricate. This is the case of classes included in matter and life strata as well as of the artifacts class. Except for continuum bodies, which contain phenomena that can be considered within the quality type, the rest will be included in the material entity type. As for the class energy we follow the "future directions" of BFO as pointed by Smith et al. (2015, 6): "[p]ortion of energy potentially to be treated as child of material entity."

The remaining ILC classes in the dependent continuant column of Table 1 contain phenomena that do not fit into the quality entity type, such as continuum bodies, but depend specifically or generically on the existence of some material entity to exist. Some could be considered what Smith (2016) calls a "total complex of dispositions" of which, according to the author, the English language, the law, the price system, the Christian

2 The Information Artifact Ontology and the Ontology for Biomedical Investigations provide the resources to deal with numerical measurement results and with certain other mathematical entities. 
faith are examples. In any case, when it comes to "social objects," the ontological analysis becomes more complex.

Finally, the phenomena of the two classes of the information stratum would be of the entity type spatiotemporal region. The spacetime layer will be the only one at the formal level of the BFO entities.

\subsection{Discussion}

When comparing the units of the two systems the most obvious difference can be attributed to the purely formal level of abstraction of the BFO. This characteristic is decisive for the gap between the units of the two systems. Another relevant difference is the grouping of phenomena in some ILC classes which, in the BFO approach, represent complex compounds of entities. In technologies class, for example, we can find the technological systems themselves, that would be a type of dependent continuant, and occurrent entities such as fishing and mining. Nevertheless, besides the spacetime class alignment, another similarity can be pointed out. One of the reasons for mathematical entities not integrating BFO is that they stand outside of space and time. In ILC, the class forms, that encompass those entities, is the only one that is not included in the nature "superclass" that encompasses, besides the other classes of the information strata, all classes belonging to the matter, life and mind levels.

Another similarity between the two systems will be in terms of the instrumental use of the concepts, although with different theoretical and, allegedly, procedural positions. In ILC, concepts are understood as units of meaning that, validated by science, constitute the intersubjective component of phenomena. These will be located in the ontological dimension that can be publicly represented. In BFO the concepts are used in the Aristotelian tradition of ontological analysis. In this perspective the ontology represents the real-world entities, and their relations, and not the concepts used to access them. These are understood as being meanings agreed upon by a group of responsible people and, in the words of Klein and Smith (2010, 437), "'partitive' and 'associative relations' (such as part_of or causes) are not appropriate for concept systems - since they are not relations which hold between meanings." For those authors, only the narrower than relationship and its inverse should be used to link concepts. One may wonder which model is the most consistent in the ontological approach to knowledge organization.

\subsection{Final remarks}

The initial state of the study leaves several questions open that we intend to address in the future. Among these, is the deepening of the relationship between conceptual and representation systems, the nature and constitution of concepts and the distinction between epistemological and ontological knowledge. We consider them to be complex issues, but of great relevance for an area of study that intends to organize knowledge.

\section{References}

Arp, Robert, Barry Smith, and Andrew D. Spear 2015. Building Ontologies with Basic Formal Ontology. Cambridge, Massachusetts: The MIT Press.

Bardin, Laurence. 2011. Análise de Conteúdo. São Paulo: Almedina.

Dahlberg, Ingetraut. 2017. "Why a New Universal Classification System." Knowledge Organization 44: 65-71. 
Gnoli, Claudio. 2008. "Ten Long-Term Research Questions in Knowledge Organization." Knowledge Organization 35: 137-149.

Gnoli, Claudio. 2018. "Classifying Phenomena, Part 4: Themes and Rhemes." Knowledge Organization 45: 43-53.

Hjørland, Birger. 2010. "Concepts, Paradigms and Knowledge Organization.” In Paradigms and Conceptual Systems in Knowledge Organization: Proceedings of the Eleventh International ISKO Conference 23-26 February 2010, Rome, Italy, edited by Claudio Gnoli and Fulvio Mazzocchi. Advances in knowledge organization 12. Würzburg: Ergon Verlag, 38-42.

Klein, Gunnar O. and Barry Smith. 2010. "Concept Systems and Ontologies: Recommendations for Basic Terminology." Transactions of the Japanese Society for Artificial Intelligence 25, no. 3: 433-441.

Kleineberg, Michael. 2017. "Integrative Levels.” Knowledge Organization 44: 349-380.

Kleineberg, Michael. 2020. "Classifying Perspectives: Expressing Levels of Knowing in the Integrative Levels Classification.” In Advances in knowledge organization 17.

Kuckartz, Udo. 2014. Qualitative Text Analysis: A Guide to Methods, Practice and Using Software. translated by A. McWhertor. London: SAGE Publication.

Machado, Luís, Mauricio B. Almeida, and Renato Rocha Souza. 2020. "What Researchers Are Currently Saying About Ontologies: A Review on Recent Web of Science Articles." Knowledge Organization 47: 199-219.

Poli, Roberto and Leo Obrst. 2010. "The Interplay Between Ontology as Categorial Analysis and Ontology as Technology." In Theory and Applications of Ontology: Computer Applications, edited by Roberto Poli, Michael Healy, and Achilles Kameas. Dordrecht: Springer, 1-26.

Ridi, Riccardo. 2016. "Phenomena or Noumena? Objective and Subjective Aspects in Knowledge Organization." Knowledge Organization 43: 239-253.

Simões, Maria da Graça, M. Cristina V. de Freitas, and Blanca Rodríguez-Bravo. 2016. "Theory of Classification and Classification in Libraries and Archives: Convergences and Divergences." Knowledge Organization 43: 530-538.

Smith, Barry et al. 2015. Basic Formal Ontology 2.0: Specification and User's Guide. https://github.com/BFO-ontology/BFO

Smith, Barry. 2016. Analytic Metaphysics 4: Ontological Realism. Buffalo: National Center for Ontological Research.

Szostak, Rick, Claudio Gnoli, and María López-Huertas. 2006. Interdisciplinary Knowledge Organization. Cham: Springer. 